\title{
Frontières
}

\section{Le système politique belge et la gestion des questions éthiques}

\section{Le cas de l'euthanasie}

\section{Nathalie Schiffino et Catherine Bert}

Volume 20, numéro 1, automne 2007

La « bonne mort»

URI : https://id.erudit.org/iderudit/017946ar

DOI : https://doi.org/10.7202/017946ar

Aller au sommaire du numéro

\section{Éditeur(s)}

Université du Québec à Montréal

ISSN

1180-3479 (imprimé)

1916-0976 (numérique)

Découvrir la revue

Citer cet article

Schiffino, N. \& Bert, C. (2007). Le système politique belge et la gestion des questions éthiques : le cas de l'euthanasie. Frontières, 20(1), 40-46.

https://doi.org/10.7202/017946ar
Résumé de l'article

La loi du 28 mai 2002 dépénalisant l'euthanasie place la Belgique dans une situation exceptionnelle par rapport à la plupart des autres démocraties occidentales. Cette contribution montre quels traits majeurs du système politique belge permettent d'expliquer l'adoption de décisions publiques autorisant le dépassement de seuils normatifs à propos des enjeux éthiques qui impliquent des comportements éminemment individuels tel le recours à l'euthanasie. L'article procède également à une analyse des dispositions prévues par la loi du 28 mai 2002, de leur application par les médecins et des questions éthiques qu'elles soulèvent. Les débats au sens politique et échanges de paroles au sens éthique, les libertés mises en oeuvre et le statut de la norme sont au centre de cette réflexion. 


\section{Résumé}

La loi du 28 mai 2002 dépénalisant l'euthanasie place la Belgique dans une situation exceptionnelle par rapport à la plupart des autres démocraties occidentales. Cette contribution montre quels traits majeurs du système politique belge permettent d'expliquer l'adoption de décisions publiques autorisant le dépassement de seuils normatifs à propos des enjeux éthiques qui impliquent des comportements éminemment individuels tel le recours à l'euthanasie. L'article procède également à une analyse des dispositions prévues par la loi du 28 mai 2002, de leur application par les médecins et des questions éthiques qu'elles soulèvent. Les débats au sens politique et échanges de paroles au sens éthique, les libertés mises en œuvre et le statut de la norme sont au centre de cette réflexion.

Mots clés: euthanasie - Belgique politiques publiques - éthique.

\begin{abstract}
The Act of May 28, 2002 decriminalizing euthanasia puts Belgium in a unique position compared with most other Western democracies. Our contribution aims at explaining the main political traits allowing a permissive Belgian public policy regarding euthanasia. The adoption of such a collective norm is tightly linked with individual behaviors and it derivates from ethical choices at a personal but also societal level of decision. The article also carries out an analysis of the provisions of the Act of May 28, 2002, enforcement by doctors and the ethical issues they raise. Politically oriented debates and exchanges surrounding ethical issues, freedoms implemented and the topic of normalization are at the heart of this reflection.
\end{abstract}

Keywords: euthanasia - Belgium public policies - ethics.

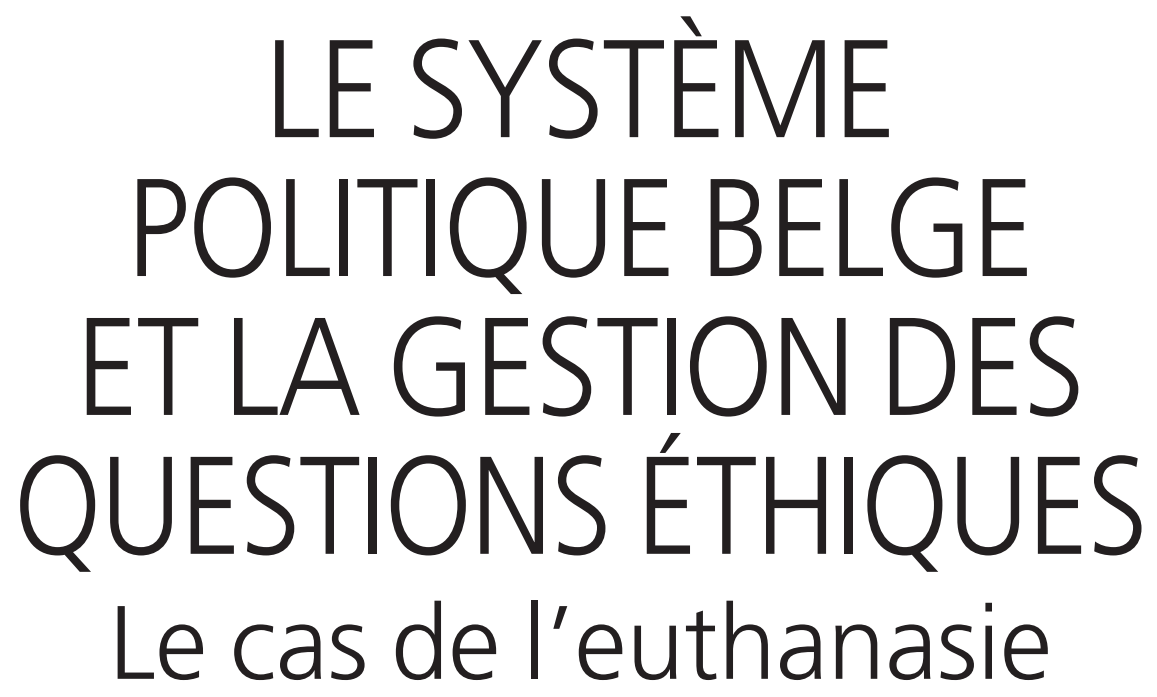

Dr Nathalie Schiffino, Ph. D., politologue, professeure de science politique, FUCaM, Département des sciences politiques, sociales et de communication.

Catherine Bert, M.S., philosophe, assistante et maître de conférences aux FUNDP, Faculté de droit.

\section{L'EUTHANASIE SOUS L'ANGLE DE VUE POLITIQUE ET PHILOSOPHIQUE}

La Belgique partage avec les PaysBas la caractéristique d'avoir dépénalisé l'euthanasie ${ }^{1}$. Dans d'autres États unitaires (l'Espagne, le Danemark) ou fédéraux (la Suisse, l'Australie, les États-Unis d'Amérique et plus spécifiquement l'Oregon), des débats et des décisions ont également alimenté le dilemme entre les avancées médico-scientifiques et le droit des personnes de pouvoir choisir le moment de leur propre mort. Dans les cas belge et néerlandais, ce qui frappe, c'est le choix d'un instrument fort de politiques publiques, la loi, pour trancher un enjeu éthique sensible, l'euthanasie, dans le sens du respect de l'autonomie des soignés, de leurs proches et des équipes soignantes. Après débats, la norme est donc celle qui privilégie l'autonomie individuelle.

Notre contribution s'articule autour de trois notions: débats, normes et libertés. Celles-ci se situent à l'intersection des sphères politique et éthique. La dépénalisation de l'euthanasie en Belgique est la résultante d'interactions entre pratiques médicales, enjeux éthiques et débats politiques, assorties de décisions publiques dans une conjoncture propice. En effet, les pratiques médicales d'euthanasie étaient officieusement déjà présentes bien avant la loi du 28 mai 2002, et elles suscitaient des questionnements éthiques. C'est à la faveur de la coalition gouvernementale laïque formée en 1999 que le problème socialement construit depuis les années 1980 a été mis à l'agenda. La loi de 2002 met en place des procédures nouvelles, qui laissent poindre de nouvelles questions dont le ressort éthique est déterminant et qui inviteraient à de nouvelles décisions politiques. Par de tels dépassements, à l'instar d'autres problématiques, l'euthanasie se situe donc bien dans une perspective cyclique des politiques publiques.

La première partie de l'article envisage, sur un plan macroscopique, comment l'adoption d'une norme relative à un enjeu moral est débattue afin que la décision politique s'inscrive dans le sens $\mathrm{du}$ respect (voire de la promotion) de la liberté de conscience. Dans la deuxième partie, le fonctionnement du système politique belge est illustré par l'analyse des pratiques belges en matière d'euthanasie à la lumière de la loi du 28 mai 2002. 
En effet, les rapports de la Commission fédérale de contrôle et d'évaluation de l'euthanasie, créée par la loi, ainsi que des enquêtes comparatives à l'échelle européenne, permettent de dresser un premier bilan de l'application de la loi. On verra quels éléments significatifs en retenir, en termes de pratiques médicales, mais aussi et surtout d'enjeux éthiques liés à l'échange de paroles, d'exercice de la liberté et de statut de la norme. Dans la conclusion, on s'attachera à synthétiser en quoi la cohabitation entre la loi et l'individualité des expériences est importante sur le plan éthique, et symptomatique du fonctionnement du système politique belge. En effet, par définition, la loi est un ensemble de règles obligatoires prises par et pour la collectivité. Il est donc important - et singulièrement pour des problématiques comme celle de l'euthanasie (c'est-à-dire de la «bonne mort» étymologiquement parlant) - d'envisager comment les principes définis collectivement sont appliqués par les individus et quelles réflexions ces principes nourrissent sur le plan éthique.

\section{ÉVOLUTION DU SYSTÈME POLITIQUE BELGE À PROPOS DE LA PROBLÉMATIQUE DE L'EUTHANASIE}

\section{L'EUTHANASIE :}

\section{DE LA RES PRIVATA À LA RES PUBLICA}

Comment un enjeu moral et privé est-il politisé ? Le lien entre euthanasie et politique est passionnant pour qui s'intéresse au fonctionnement de nos démocraties occidentales, et singulièrement à l'évolution de leurs modes opératoires, notamment sous la pression tant, d'une part, des innovations (bio)technologiques et médicales que, d'autre part, de la mutation des valeurs. Comment les démocrates, élus et citoyens, débattent-ils en vue de décisions publiques à propos d'une problématique l'euthanasie - qui relevait, y a quelques décennies encore, de la sphère privée ${ }^{2}$.

Différentes approches relevant des sciences politiques ont abordé la question de l'euthanasie et de sa dépénalisation en Belgique: de manière non exhaustive, citons ici la démarche comparative et l'ancrage dans la théorie des partis politiques (Green-Pedersen, 2007) ; l'analyse des rapports de pouvoir entre loi, politique et biomédecine (Muraille, 2000; Baum, 2001); l'approche socio-historique insistant sur les faits, les acteurs et leurs interactions, ce qui comprend plusieurs descriptions historiques $\mathrm{du}$ processus de régulation (Wautier, 2000). À côté de ces nombreuses contributions, la combinaison de deux dimensions retient ici plus particulière-

ment l'attention et permet de préciser un angle d'approche spécifique: la mise en œuvre de la démocratie et le passage d'un fait social - en l'occurrence l'euthanasie de la sphère privée à la sphère publique.

Quelles dynamiques sont instaurées dans les débats autour des normes à établir concernant les libertés des individus vivant ensemble au sein d'une société? En quoi sont-elles démocratiques? Par la mise

\section{CE N'EST PAS SEULEMENT}

\section{LA PERSONNE AUX PRISES}

AVEC SES SOUFFRANCES QUI

EST CONCERNÉE, MAIS

L'ENSEMBLE DE LA SOCIÉTÉ

DANS LAQUELLE CETTE

PERSONNE EST INTÉGRÉE.

en œuvre de débats? Par les procédures utilisées: discussions, recours à l'arène législative dotée de la légitimité du pouvoir par élections, auditions voire participation de groupes et de citoyens représentatifs au sein de la société civile? Par la pluralité des acteurs impliqués dans le processus de décision? Par les interactions entre des acteurs aux positions antagonistes? Par le consensus qui peut émerger des débats ou, au contraire, par le fait que celui-ci puisse aboutir à des non-décisions ${ }^{3}$ ?

Ces questions sont pertinentes dans la mesure où un acte privé (celui d'anticiper la mort à cause des souffrances infligées par une maladie dont l'issue est inéluctable) relève en définitive du champ public. Ce n'est pas seulement la personne aux prises avec ses souffrances qui est concernée, mais l'ensemble de la société dans laquelle cette personne est intégrée. En soi, l'euthanasie est un fait social, parce qu'elle implique des tiers. En effet, tout fait devient social dès lors qu'il est significatif pour d'autres et se place donc dans un réseau d'interactions (Rezsohazy, 1984, p. 24-26). La mort ne se réduit pas au seul phénomène biologique, mais aux conceptions que la société en développe et aux comportements sociaux qu'elle élabore en conséquence.

C'est donc bien à un problème social auquel on se trouve confronté, non pas d'un point de vue normatif, mais selon l'acception du terme en sciences politiques et, de manière plus générale, en sciences sociales. En effet, il y a problème social quand il existe un écart entre l'état actuel et l'état désiré d'une situation. Cet écart suscite une insatisfaction, provoque un besoin qui est détecté directement ou par des manifestations extérieures, et une recherche de solutions collectives est alors enclenchée (Knoepfel et al., 2001).

\section{LE SYSTÈME POLITIQUE \\ AUX PRISES AVEC LA DÉFINITION \\ DE NORMES ÉTHIQUES}

La dynamique qui est ainsi mise en branle met en évidence la politisation de l'euthanasie, c'est-à-dire non seulement sa prise en charge dans les axes programmatiques des partis politiques, mais bien plus, son insertion dans la gestion de la res publica. Pourquoi et comment l'euthanasie est-elle, à un moment donné de l'évolution d'une société et de son système politique, mise en débat et, le cas échéant, soumise à des décisions publiques? En Belgique, la question prend une ampleur particulière avec la mise en œuvre d'une politique publique dépénalisant l'euthanasie, contrairement à des pays comme la France où les débats publics n'ont pas débouché jusqu'ici sur un tel instrument régulatoire.

Les trente dernières années ont accéléré la gestion politique d'enjeux moraux (morality issues dans les termes de Smith et al., 2003) tels que l'euthanasie (dépénalisée en Belgique en 2002) mais aussi l'interruption volontaire de grossesse (dépénalisée en Belgique par la loi du 3 avril 1990), le mariage entre personnes de même sexe (permis par la loi du 13 février 2003), la procréation médicalement assistée (ayant fait l'objet de régulations progressistes en 1999 puis des lois du 11 mai 2003 et du 6 juillet 2007). Cette situation s'explique en partie par les progrès considérables réalisés en médecine et les questions bioéthiques qu'ils soulèvent, soulignant l'importance du lien entre science (développement de nouvelles techniques et pratiques) et politique (définition de programmes publics pour réguler les innovations) ${ }^{4}$.

De façon générale, la mise à l'agenda public peut être provoquée par différents catalyseurs. La politisation peut être le fruit de pressions de la part de la société civile. Cependant, en Belgique, il est intéressant de constater que, certes, des associations médicales et des groupes d'intérêt axés sur la problématique de l'euthanasie existent, mais qu'ils ne sont pas à l'origine de l'effort législatif ${ }^{5}$. Le développement d'affaires judiciaires n'est pas davantage un élément déterminant: très peu de procès publics ont eu lieu (Wautier, 2000, p. 12). Une médiatisation préalable des enjeux de l'euthanasie n'est pas davantage un facteur explicatif significatif. En réalité, 
l'impulsion de la régulation en matière d'euthanasie revient principalement aux partis politiques.

Elle s'explique à la fois par une tendance lourde (l'existence de clivages depuis la naissance de l'État belge en 1830) et un facteur conjoncturel (la formation d'une coalition excluant les partis sociaux-chrétiens francophone et flamand en 1999). En effet, pour comprendre la régulation publique de l'euthanasie en Belgique, il faut impérativement prendre en compte l'existence de clivages tels qu'ils ont été définis par S. Rokkan (1970). Jusque dans les années $1960^{6}$, l'opposition entre catholiques et partis laïcs a structuré la prise de décisions politiques sur des enjeux spécifiques (l'organisation des enterrements, le développement de l'enseignement, etc.) mais aussi plus généraux (l'extension du droit de vote, la structuration des forces syndicales, etc.). Malgré le processus de sécularisation constaté dans tous les pays européens (voir Eurobaromètres et European Values Studies), les positionnements éthiques, notamment à propos de l'euthanasie, sont intrinsèquement liés aux lignes emblématiques des partis politiques.

En fonction des circonstances, les partis politiques (dont le poids décisionnel est prépondérant dans le système décisionnel en Belgique; voir Seiler, 2000) peuvent donc choisir de se positionner ou non sur des enjeux éthiques comme l'euthanasie. En 1999, et pour la première fois depuis la fin de la Deuxième Guerre mondiale, une coalition gouvernementale a rassemblé l'ensemble des partis laïcs (représentant le libéralisme politique, le socialisme démocratique et l'écologie politique) et a laissé dans l'opposition les partis sociauxchrétiens. L'adoption de normes dans le domaine éthique, entre autres à propos de l'euthanasie, s'est faite à la faveur de cette fenêtre d'opportunités.

LA GESTION DES LIBERTÉS INDIVIDUELLES PAR LE SYSTĖME POLITIQUE BELGE

Comme pour d'autres problématiques éthiques en Belgique, l'euthanasie était régulée par défaut avant 2002. Par là, il faut entendre qu'elle n'était pas mentionnée explicitement dans un corpus juridique. Passée sous silence, elle tombait néanmoins sous le coup du Code pénal en vertu duquel, si des plaintes étaient déposées contre des personnes ayant pratiqué des actes d'euthanasie, il était possible de les poursuivre en justice et, le cas échéant, de les condamner. La principale protection à l'égard des poursuites judiciaires était donc le secret médical (le silence prend encore ici tout son sens). Concrètement, peu de dénonciations ont été recensées et

LE PATIENT, ANTICIPATIVEMENT OU PAS, ET SES PROCHES

ONT LA LIBERTÉ DE CHOISIR L'EUTHANASIE. LE SOIGNÉ A ÉGALEMENT

LA LIBERTÉ DE REFUSER UN TRAITEMENT DE LA SOUFFRANCE, Y COMPRIS PALLIATIF. LE MÉDECIN ET L'ÉQUIPE MÉDICALE ONT LA LIBERTÉ D'ACCEPTER OU DE REFUSER DE PRATIQUER LES ACTES D'EUTHANASIE SUR LEURS PATIENTS.

peu d'affaires ouvertes en Justice. La loi de 2002 sur l'euthanasie a consisté, à l'instar de ce qui a été décidé en matière d'avortement en 1990, à dépénaliser l'acte.

Ce choix de régulation publique (la dépénalisation) trouve ici une explication; les décideurs l'ont privilégiée parmi l'éventail d'instruments normatifs à leur disposition, entre l'interdiction et l'autorisation. Le choix de cette norme politique implique que si la Commission fédérale de contrôle et d'évaluation de l'euthanasie chargée d'examiner les dossiers d'euthanasie introduits a posteriori par les médecins - estime que l'un d'eux a pratiqué l'acte de manière abusive, sans respecter les conditions fixées par la loi de 2002, elle peut le renvoyer devant la Justice. À ce jour, aucun cas d'espèce n'est connu.

Cette situation est symptomatique de la façon dont le système politique belge gère les libertés individuelles (qu'il est tentant de qualifier de privées) dans l'arène publique. En effet, sans autoriser explicitement l'euthanasie, les décideurs politiques (il faut entendre par là le législateur mais également les partis politiques représentés par la technique proportionnelle au sein du Parlement) accordent plusieurs libertés aux acteurs de la société civile.

Le patient, anticipativement ou pas, et ses proches ont la liberté de choisir l'euthanasie. Le soigné a également la liberté de refuser un traitement de la souffrance, y compris palliatif ${ }^{7}$. Le médecin et l'équipe médicale ont la liberté d'accepter ou de refuser de pratiquer les actes d'euthanasie sur leurs patients. Il est également intéressant de noter que le vote de la loi dépénalisant l'euthanasie a été réalisé en respectant la liberté de conscience des parlementaires. Ce fait n'est pas anodin dans une particratie où la discipline de vote est imposée par les partis politiques à leurs élus sur la plupart des questions traitées au Parlement, à l'exception des questions éthiques.

L'insistance sur les principes d'autonomie, de libre disposition de soi, de liberté de conscience doit être comprise au regard de la prégnance du clivage philosophicoreligieux en Belgique. Dans cette perspective, tout problème social tel qu'il est défini ci-dessus relève d'une construction sociale dépendant entre autres des conceptions culturelles. Cette construction sociale s'oppose à une vision positiviste obéissant à une rationalité technique : selon celle-ci, la primauté des savoirs scientifiques et le rôle des experts (les médecins dans le cas de l'euthanasie) prédomineraient dans la définition des causes et des solutions à apporter aux situations problématiques, sous le couvert de données objectivables comme des statistiques.

DÉBATTRE : LE NEUD

DU SYSTĖME POLITIQUE BELGE

Deux décennies de débats contradictoires ont précédé la régulation de l'euthanasie en Belgique. Malgré le blocage décisionnel des sociaux-chrétiens au pouvoir, des propositions de lois ont été déposées depuis les années 1980. Jusqu'en 2002, aucune enquête statistique globale ne permettait d'objectiver les pratiques. L'échange d'arguments reposait sur la fonction de la norme, la dignité des patients, le rôle des équipes médicales, etc., autant de composantes subjectives et qualitatives de l'euthanasie.

Le système social belge dans son ensemble cultivait le paradoxe de couvrir des pratiques réalisées sous le sceau du secret médical et de porter dans l'arène publique des discours attestant des actes officieux. Un des mérites de la loi de 2002 sur l'euthanasie est de libérer davantage les discussions, qu'elles concernent le public, les soignés, les équipes soignantes sur, par exemple, les possibilités d'euthanasie active dans des situations désespérées (Englert et al., 2005, p. 151).

Dans une optique plus générale, le propre de la démocratie est d'ouvrir de tels espaces de parole, des débats contradictoires, afin que les choix de société reflètent avec le plus de représentativité possible la diversité de la société. C'est un des traits de la démocratie, qu'elle soit définie par J. Habermas (1984) dans l'optique de l'impératif délibératif, ou par les théories contemporaines détaillant les modalités concrètes de participation citoyenne (Revue suisse de science politique, 2004). 
D'un point de vue épistémologique, considérer les débats publics comme un «nœud» leur confère différentes propriétés (Schiffino, 2003). Cela implique que les débats sur des enjeux politisés peuvent poser problème, mais aussi qu'ils peuvent être démêlés et, en tout état de cause, qu'ils s'inscrivent au centre du fonctionnement politique. Les longs travaux parlementaires sur l'euthanasie, incluant l'audition d'experts et de témoins de la société civile, n'échappent pas à cette logique et relèvent de la logique consociationnelle mise en évidence par A. Lijphart (1999) au sujet de la Belgique.

Par démocratie consociative, ou consensuelle, on entend une démocratie dans laquelle le pouvoir est partagé entre les principaux groupes qui composent la société. La démocratie fonctionne alors sur base de compromis entre les acteurs politiques mais aussi entre les groupes qui représentent les différentes facettes de la réalité nationale: groupes religieux, communautés linguistiques, groupements patronaux, etc. Au regard des dix critères sous-tendant la typologie de Lijphart sur les régimes consensuels et majoritaires, la Belgique constitue une illustration caractéristique de la démocratie de consensus.

À première vue, le cas de l'euthanasie semblerait contredire cette assertion. En effet, la législation a été adoptée alors que les sociaux-chrétiens se trouvaient dans l'opposition. Le consensus pouvait apparaître incomplet: les principaux acteurs politiques de blocage se trouvaient minorisés; une logique de bloc à bloc, partis laïcs versus chrétiens, dans le pur sens du clivage défini par S. Rokkan, prévalait. En réalité, le processus de décision a attesté de la fiabilité du jeu parlementaire, par la prise en compte des propositions de lois et des amendements à la loi avancés par la minorité sociale chrétienne.

\section{ÉVOLUTION DES PRATIQUES ET DES ENJEUX ÉTHIQUES LIÉS À L'EUTHANASIE EN BELGIQUE}

On le voit, les conditions de possibilité du processus de dépénalisation de l'euthanasie sont le fruit d'interactions entre les décisions politiques, les pratiques médicales et les expériences citoyennes. La dépénalisation de l'euthanasie constitue un choix politique et sociétal qui situe la «bonne mort» à un niveau normatif explicite. En ce sens, la loi du 28 mai 2002 permet une meilleure prise en charge de certaines situations de fin de vie. Néanmoins l'évaluation des pratiques mises en place depuis son entrée en vigueur soulève de nouveaux problèmes. Certains d'entre eux font l'objet de nouvelles réglementations ${ }^{8}$. D'autres, par contre, laissent apparaître des enjeux éthiques.
ÉVALUATION DES ACTES

DE FIN DE VIE AU REGARD

DE LA LOI BELGE DU 28 MAI 2002

Récemment menée à travers quatre pays européens, une enquête a permis de mettre en évidence quelques résultats significatifs à propos des pratiques existantes en Belgique, en matière de fin de vie, depuis l'entrée en vigueur de la loi (Jooken et Sermeus, 2007) ${ }^{9}$. L'analyse de ces pratiques porte sur la nature des actes de fin de vie ainsi que sur la signification attribuée à ces actes par le soigné et par le soignant. Les résultats de l'enquête permettent de pointer les décalages qui subsistent entre les normes, les représentations individuelles et les pratiques soignantes. On constate par exemple que sur 100 demandes d'euthanasie, 26 sont effectivement pratiquées ou encore que $88 \%$ des médecins interrogés disent avoir pratiqué au moins une fois un acte d'interruption de vie, compris au sens large du terme.

\section{La loi belge du 28 mai 2002}

La loi belge relative à l'euthanasie énonce les différentes conditions qui justifient cet acte. Le patient doit être majeur (ou mineur émancipé) et en état d'exprimer sa volonté. La demande doit se faire de manière volontaire, réfléchie et répétée, sans pression extérieure. La maladie doit être grave et incurable et entraîner des souffrances physiques ou mentales constantes, insupportables et impossibles à soulager. Le médecin doit envisager avec le patient, son état de santé, son espérance de vie, les possibilités thérapeutiques et palliatives et leurs conséquences. Au terme de ces discussions, le médecin doit arriver avec le patient à la conclusion qu'il n'existe pas d'autre solution raisonnable et que la demande du patient est entièrement volontaire. Il doit également consulter un confrère indépendant et compétent quant à la pathologie concernée, qui s'entretiendra lui aussi avec le patient. Si une équipe médicale est en contact régulier avec le patient, il doit s'entretenir avec celle-ci, ou du moins avec des membres de celle-ci. Si le patient le souhaite, le médecin doit aussi s'entretenir avec les proches du patient que celui-ci aura désignés. En outre, si le médecin estime que le décès n'interviendra pas à brève échéance, il doit consulter un deuxième médecin et laisser s'écouler au moins un mois entre la demande écrite du patient et l'euthanasie. La demande du patient doit faire l'objet d'un document rédigé, daté et signé par le patient lui-même ou par une personne majeure de son choix et n'ayant aucun intérêt matériel à son décès. Le patient peut à tout moment révoquer sa demande.
Ces conditions remplies, l'euthanasie peut être pratiquée. Le médecin doit ensuite compléter un document d'enregistrement et le renvoyer à la Commission fédérale de contrôle et d'évaluation de l'euthanasie.

La nature de la demande d'euthanasie

La notion de demande requiert une approche nuancée. On peut distinguer: 1) la demande de ne pas entamer un traitement qui pourrait allonger la vie, 2) la demande d'arrêter un traitement, 3) la demande d'augmenter fortement la dose de médicaments contre la douleur et les symptômes au point d'accroître le risque d'un décès plus rapide, 4) la demande de suicide assisté, 5) la demande d'euthanasie, 6) la demande de sédation palliative profonde accompagnée de l'arrêt d'alimentation parentérale et de l'hydratation. L'euthanasie apparaît ainsi comme un acte parmi les autres actes de fin de vie.

Selon les résultats de l'enquête, la demande de pratiquer une euthanasie (administration d'un médicament létal) est dominante en Belgique. Par contre, dans les autres pays (l'Espagne, l'Italie et le Portugal où il n'existe pas de loi dépénalisant l'euthanasie), la demande est davantage formulée comme demande d'aide au suicide (prescription ou fourniture d'un médicament létal).

\section{La motivation de la demande}

d'euthanasie

Les demandes d'interruption de vie sont plus nombreuses lorsque les soins (démarrage ou poursuite de traitement) n'assurent plus une qualité de vie.

La première raison invoquée pour justifier la demande d'euthanasie ou d'aide au suicide est la douleur insupportable (voir Englert et al., 2005, p. 148). D'autres raisons interviennent telles que la peur de nouvelles détériorations physiques, la perte du sens de la vie, la peur de perdre sa dignité ou l'angoisse de la douleur à venir.

En Belgique, en vertu de la réglementation en vigueur, une corrélation a pu être établie entre les indices de qualité de fin de vie et de qualité de la mort. Ceux-ci sont plus élevés lorsque la mort intervient, soit avant, soit aux environs du «moment naturel ${ }^{10}$. Cet indice de qualité disparaît lorsque l'acte de fin de vie est posé après le moment naturel et lorsque le patient a subi un acharnement thérapeutique.

Notons qu'aucune différence significative n'apparaît dans le type de demandes entre les patients bénéficiant de soins palliatifs et les patients bénéficiant d'autres soins. L'enquête ne permet toutefois pas de préciser si la demande intervient plus 
tardivement de la part de patients bénéficiant de soins palliatifs.

Selon ces données, la pratique de l'euthanasie permet aux patients comme aux proches de retrouver le sens premier du mot: une mort douce et sans souffrance. L'euthanasie comme l'aide au suicide sont bien souvent associées à l'exigence d'une meilleure qualité des derniers moments.

\section{De la demande à la réponse}

L'existence d'une loi relative à l'euthanasie amène à insérer celle-ci davantage dans le processus de prise en charge du soigné. En effet, l'euthanasie s'inscrit alors dans la continuité des soins qui visent à soulager la douleur et qui impliquent parfois de ne pas entamer de nouveau traitement, ou d'arrêter le traitement, ou d'augmenter fortement les doses de médicaments, ou encore de donner la mort.

Néanmoins, les chiffres qui attestent du rapport entre, d'une part, la demande d'euthanasie et, d'autre part, la réponse à cette demande sont interpellants. Sur 100 demandes d'euthanasie, 26 sont déclarées et pratiquées. Une première interprétation de ces données invite à penser que la réponse à une demande d'euthanasie n'est pas systématique. Par contre, les soignants répondent plus favorablement, par exemple, à une demande d'arrêt de traitement $(98 \%)$. Une seconde interprétation du décalage entre le nombre de demandes et le nombre d'euthanasies effectivement pratiquées et déclarées (26\%) peut également être donnée eu égard à une problématique entourant le document d'enregistrement.

\section{Le document d'enregistrement}

d'une euthanasie

Il pourrait s'avérer pertinent de mener une enquête comparant le nombre d'euthanasies pratiquées et ayant fait l'objet d'une déclaration avec le nombre d'euthanasies pratiquées n'ayant pas fait l'objet d'une déclaration. Ce type d'enquête existe aux Pays-Bas, mais pas en Belgique.

Les raisons pour lesquelles le médecin ne remplit pas le document d'enregistrement (la déclaration) peuvent être liées au choix de la technique d'euthanasie. Le rapport de la Commission fédérale de contrôle et d'évaluation de l'euthanasie (2006) ne signale, par exemple, aucun décès par sédation terminale comme un acte d'euthanasie. En effet, cette technique peut être interprétée comme le moyen d'assurer la fin de vie dans l'inconscience, sans avoir pour but d'entraîner la mort, cette dernière survenant comme l'évolution naturelle de la maladie. Or cette interprétation est contestée, surtout si la technique de sédation est pratiquée en réponse à une demande d'euthanasie, sans accord sur les modalités envisagées et sans dialogue sincère (Bilsen, Cohen et Dilens, 2007, p. 3) ${ }^{11}$.

Un autre décalage dans les chiffres doit également être pris en considération. Dans son deuxième rapport, la Commission fédérale de contrôle et d'évaluation de l'euthanasie mentionne les chiffres suivants, pour les années 2004 et 2005: 86\% des déclarations sont rédigées en néerlandais et 14\% en français ${ }^{12}$. Pour expliquer cet écart entre le nombre de documents d'enregistrement rédigés en français et en néerlandais, elle émet des hypothèses telles qu'une attitude socioculturelle face à la mort qui serait différente au Nord et au Sud du pays, des informations sur la législation relative à l'euthanasie plus largement diffusées en Flandre, des réactions différentes de la part des médecins quant à l'obligation de déclarer l'euthanasie au Nord et au Sud, ou encore une connaissance différente des produits «incontestablement létaux », les médecins néerlandophones ayant eu davantage de contact avec les médecins des Pays-Bas et leur littérature scientifique sur le sujet.

\section{LES ENJEUXÉTHIQUES}

SOULEVÉS PAR LA LOI BELGE

Les réponses apportées par la loi du 28 mai 2002 soulèvent à leur tour diverses questions: l'ouverture à la discussion dans les situations de fin de vie est-elle une condition pour un échange de paroles authentiques? La conception de la liberté promue par la loi permet-elle un plus grand respect du soigné et du soignant? Les normes mises en place garantissent-elles une approche cohérente des situations de fin de vie?

\section{Les conditions de l'échange}

La loi permet aujourd'hui, à un grand nombre de patients, d'aborder la question de la fin de vie ouvertement; ce qui n'était pas toujours possible avant la législation (Bilsen, Cohen et Dilens, 2007, p. 3) ${ }^{13}$. La discussion est devenue plus libre aussi au sein des équipes soignantes. Quelle que soit l'option philosophique ou religieuse, un consensus se dégage pour admettre qu'il faut pouvoir écouter, voire apprendre à écouter, ce que le malade demande réellement lorsqu'il demande à en finir.

La difficulté d'une telle ouverture tient dans la confrontation des approches de la maladie, de la vie et de la mort à la fois radicalement différentes et fort proches. Des différences opposent le soignant, dont le but est de soigner, de guérir, et le soigné, qui tente de donner du sens à sa maladie et à ses souffrances. Mais tous deux peuvent se rencontrer dans le partage d'expériences existentielles communes. Dans le chef du patient, différents arguments motivent le désir de mourir, comme on l'a évoqué précédemment. Il peut s'agir du sentiment pesant que l'insupportable domine le présent et le futur, de souffrances inapaisables ou encore du sentiment de perte de dignité. L'ouverture à la parole signifie aussi parler des craintes et des désirs du malade, laisser une place aux proches et les soutenir dans leur tâche d'accompagnement. Face à la demande, le soignant doit entendre la parole du patient - et éventuellement de sa famille -, et discerner ce qui est demandé et ce que cette parole évoque pour lui. La demande d'en finir le renvoie à des mécanismes parfois inconscients d'échec, d'impuissance ou de culpabilité.

Comme le soigné, le soignant est renvoyé à sa propre vulnérabilité face à la mort: de part et d'autre, il s'agit de pouvoir humblement l'accepter. L'ouverture à la parole n'est authentique qu'à la condition que chaque parole puisse être écoutée et respectée dans ce qu'elle exprime réellement. Or une loi peut-elle être un facteur facilitant le développement de telles attitudes? Certes la loi facilite l'ouverture à la parole, mais les conditions de l'échange restent fort peu abordées. La disponibilité des soignants, pour écouter le malade ou pour se former, est bien souvent insuffisante. Les conditions concrètes du travail hospitalier (personnels réduits, lourdeurs des tâches administratives, impératifs de rentabilité, etc.) ne sont, par exemple, aucunement prises en compte.

\section{Une conception de la liberté}

La dépénalisation de l'euthanasie confère, dans certaines conditions, une licéité au droit de choisir sa mort. La reconnaissance de ce droit peut être perçue comme la mise à disposition d'une liberté ultime. Mais le droit de décider quand et comment mourir peut aussi être interprété comme un droit de l'individu que l'État doit protéger parce qu'il est source d'autonomie et de dignité.

Pour les mêmes motifs, la loi belge accorde tout autant de poids à la liberté du médecin. Celui-ci n'est en aucun cas tenu de pratiquer une euthanasie. Cette approche intransigeante de la liberté reste problématique pour penser la continuité de la relation de soins. La dimension humaine de la relation se dilue dans une norme dont la forme est plus déterminante que le contenu. Au nom de l'autonomie et de la dignité, il arrive en effet que le soignant et le soigné se retranchent dans une position désormais normative qui compromet la qualité et la continuité de la relation. Ainsi si le médecin refuse de pratiquer l'euthanasie, il doit informer le patient ou la personne de confiance en motivant son refus. Dans ces circonstances, la continuité et la 
qualité de la relation sont compromises. En outre et si le patient le demande, il est tenu de communiquer le dossier médical au médecin désigné par le patient. La continuité des soins reste possible, mais des dérives apparaissent. Certaines institutions de soins, dans lesquelles on pratique l'euthanasie, reçoivent les patients qui ont dû faire face à un refus dans une autre institution et sont confrontées à un nombre croissant de demandes. La concentration des demandes dans certaines institutions est acceptée par les soignants concernés. Elle est néanmoins lourde à porter pour ceux-ci, tant sur le plan émotionnel que sur le plan professionnel.

La reconnaissance du droit de choisir sa mort peut aussi être perçue comme la possibilité d'être sujet de sa mort. Dans ce contexte, elle signifie le droit d'en déterminer les conditions en cohérence avec sa propre vision du monde. Chacun est juge de sa qualité de vie, personne ne peut se substituer à lui pour l'évaluer. Cependant, reconnaître le sujet d'une telle demande est un acte qui ne peut être posé que par un autre sujet. Ni le sujet ni l'autre ne sont seuls face à la norme. Cette conception de la liberté souligne l'importance capitale des relations humaines. Les liens qui limitent notre liberté sont également regardés comme le fondement de notre ancrage dans la vie.

Le texte de la loi belge reste ambigu sur l'approche de la liberté qu'il privilégie. Or tout l'intérêt d'un tel texte est aussi de promouvoir une conception de la liberté qui renvoie à un projet de vie et à une affirmation de sa propre humanité, c'est-àdire une conception de la liberté qui soit source de dignité tant pour le soignant que pour le soigné.

\section{Le statut de la norme}

Le déplacement du seuil normatif modifie, presque inéluctablement, la façon d'appréhender les situations de fin de vie.

Une prise de position ayant pour effet de dépénaliser l'euthanasie possède le mérite de clarifier explicitement le licite et l'interdit, tout au moins d'un point de vue juridique car le niveau des pratiques peut tarder à s'adapter, comme démontré supra. Les apports majeurs d'une telle prise de position sont de responsabiliser tant le soigné que le soignant impliqués dans la demande et de reconnaître leur autonomie respective. D'autres contextes sociopolitiques privilégieront une prise de position plus nuancée. En France, par exemple, le patient en phase avancée ou terminale d'une affection grave et incurable a le droit de décider de limiter ou d'arrêter tout traitement ${ }^{14}$. Le médecin doit, dans ces circonstances, sauvegarder la dignité du mourant et assurer la qualité de sa fin de vie en dispensant des soins palliatifs ${ }^{15}$. A travers cette prise de position, la responsabilité et l'autonomie du soignant et du soigné sont intégrées dans une approche globale de la situation de fin de vie.

On peut regretter que la dépénalisation de l'euthanasie, en Belgique, n'ait pas été insérée dans une telle approche des situations de fin de vie. La loi relative aux soins palliatifs, par exemple, fait l'objet d'un texte distinct ${ }^{16}$. Or, comme le montrent les résultats de l'enquête, être pris en charge dans un service de soins palliatifs n'empêche pas dans toutes les situations une demande d'euthanasie.

En outre, le déplacement du seuil de l'interdit induit ce que d'aucuns considèrent comme un risque: l'extension de la demande. Il apparaît en effet que les mentalités évoluent, depuis que l'euthanasie est dépénalisée. La demande d'actes d'interruption de vie s'élargit. Différents projets visant à déplacer les limites de la loi actuelle font l'objet de débats. Par exemple, une proposition de loi, déposée en juillet $2004^{17}$, vise notamment à autoriser l'euthanasie pour les mineurs ${ }^{18}$ d'âge et non plus uniquement pour les patients majeurs. Cette proposition insiste également sur la nécessité d'introduire l'assistance au suicide dans la législation et de la dépénaliser au même titre que l'euthanasie. Une autre proposition de loi, déposée en décembre $2005^{19}$, vise à abroger les limitations en matière d'euthanasie en cas de démence et en cas d'inconscience prolongée (patients souffrant de la maladie d'Alzheimer ou ayant subi des hémorragies multiples, par exemple) alors que la loi actuelle concerne le patient majeur, capable et conscient.

\section{NORMES, LIBERTÉS ET DÉBATS AU CCEUR DE LA RÉFLEXION SUR L'EUTHANASIE}

On le voit, le sujet est loin d'être clos et ce, pour deux raisons au moins. Premièrement, parce que la loi et son application elles-mêmes suscitent de nouvelles interrogations sur les pratiques et leur devenir. Deuxièmement, parce que l'adoption de la loi s'inscrit dans l'essence même de la démocratie ainsi que dans les spécificités du fonctionnement du système politique belge (clivages, consensus) qui placent sans cesse les débats sur les normes au cœur de l'évolution sociale.

En 2002, l'adoption de la Loi relative à l'euthanasie a déplacé le seuil de l'interdit collectif. Ce qui était officiellement interdit, mais parfois officieusement pratiqué, est devenu licite. Les décideurs politiques ont assumé ce dépassement en interaction avec les membres de la société civile concernés au premier chef (soignés, leurs proches et les équipes soignantes) et dans le cadre du fonctionnement démocratique de base, impliquant la représentation des citoyens. La dépénalisation de l'euthanasie a été décidée dans le cadre spécifique de la définition des politiques publiques belges: clivages et particulièrement ici tension fondamentale le long de l'axe philosophicoreligieux, consensus, prééminence du rôle des partis politiques. S'agissant d'une question (bio)éthique, la discipline de partis a toutefois été assouplie pour laisser place à la liberté individuelle, tant comme modalité de décision que comme principe de base de la loi relative à l'euthanasie.

Cinq ans plus tard, l'évaluation de la mise en œuvre de la loi amène à réfléchir à d'autres types de dépassements. Premièrement, la loi cohabite avec la primauté sans cesse renouvelée de préserver la dimension humaine dans les soins de santé. En ce sens, la loi, conçue avant tout comme un outil, un instrument de politiques publiques, permet-elle de mieux préserver l'humanité des soins en matière de fin de vie? Un inconvénient de la situation actuelle pourrait résider dans la séparation des normes relatives, d'une part, à la «bonne mort » et, d'autre part, aux soins palliatifs. Cela dénote-t-il une absence de prise en charge globale de la fin de vie, dans le cadre d'une perspective cohérente?

Deuxièmement, la loi cohabite avec une grande complexité et une forte diversité des situations de fin de vie. Diverses interprétations des actes restent possibles, notamment dans le chef des médecins. Cela soulève toujours des enjeux éthiques pour les patients et leurs proches. Chaque parcours individuel pourrait être riche d'enseignements pour la collectivité. Gageons que la démocratie belge, en exploitant ses spécificités sociopolitiques comme une forte ressource d'adaptation à l'évolution constante de la société, saura y puiser une nouvelle dynamique pour continuer d'agencer au mieux les sphères publique et privée.

\section{Bibliographie}

BACHRACH, P. et M. BARATZ (1963). «Decisions and non decisions: An analytical framework », American Political Science Review, vol. 57, n 3, septembre, p. 632-642.

BAUM, M. (2001). « De la médicalisation à la juridicisation de la fin de vie », Les politiques sociales, $\mathrm{n}^{\text {os }} 1-2$.

BILSEN, J., J. COHEN et L. DILENS (2007). «La fin de vie en Europe: le point sur les pratiques médicales », Population et sociétés, $n^{0} 430$, janvier, p. 1-4.

COMMISSION FÉDÉRALE DE CONTRÔLE ET D'ÉVALUATION DE L'EUTHANASIE (2006). Deuxième rapport aux chambres législatives (2004-2005). 
COMITÉ CONSULTATIF NATIONAL D'ÉTHIQUE (2000). Fin de vie, arrêt de vie, euthanasie, Avis $\mathrm{n}^{\circ}$ 63, 27 janvier.

DELFOSSE, M. L. et C. BERT (2005). Bioéthique, droits de l'homme et biodroit: recueil de textes annotés internationaux, régionaux, belges et français, Bruxelles, Larcier, disponible en ligne: <editions.larcier.com/ livre/?GCOI $=28044100029970>, 560$ p.

ENGLERT, M., B. HANSON ET D. LOSSIGNOL (2005). «Deux années d'euthanasie dépénalisée en Belgique: comparaison avec les Pays-Bas. Premier bilan d'une unité de soins palliatifs », Revue médicale de Bruxelles, $\mathrm{n}^{\circ} 26$.

ETHICA CLINICA (2003). Dossier thématique: «L'euthanasie un an après la loi », $\mathrm{n}^{\circ} 32$.

GLORION, F. (2002). Vivre avec sa mort, Éditions de La Martinière.

GREEN-PEDERSEN, C. (2007). «The conflict of conflicts in comparative perspective: Euthanasia as a political issue in Denmark, Belgium, and the Netherlands ", Comparative Politics, vol. 39, $\mathrm{n}^{\circ} 3$.

HABERMAS, J. (1984). Theory of Communicative Action, Londres, Heinemann.

HOUZIAUX, A. (dir.) (2004). Doit-on légaliser l'euthanasie?, Paris, Les Éditions de l'Atelier / Les Éditions Ouvrières.

JOOKEN, K. et G. SERMEUS (2007). "Quand le désir de mourir l'emporte», Test Santé, $\mathrm{n}^{\circ} 77$, février-mars.

KNOEPFEL, P., C. LARRUE et F. VARONE (2001). Analyse et pilotage des politiques publiques, Bâle, Helbing \& Lichtenhahn.

LIJPHART, A. (1999). Patterns of Democracy, New Haven et Londres, Yale University Press.

MARZANO, M. (2006). Je consens, donc je suis..., Paris, Presses universitaires de France.

MONTPETIT, E., C. ROTHMAYR et F. VARONE (2006). The Politics of Biotechnology in North America and Europe: Policy Networks, Institutions and Internationalization, Lanham, Lexington Books.

MURAILLE, P. (2000). «Dans l'arc-en-ciel fédéral: l'euthanasie, sens et enjeux de l'intervention publique», La Revue nouvelle, ${ }^{\text {os }}$ 5-6.

PARSONS, W. (2003). Public Policy. An Introduction to the Theory and Practice of Policy Analysis, Cheltenham / Northampton, Edward Elgar.

REVUE SUISSE DE SCIENCE POLITIQUE (2004). vol. 10, no 4 (hiver).

REZSOHAZY, R. (1984). Théorie et critique des faits sociaux, Louvain-la-Neuve, Ciaco.

ROKKAN, S. (1970). Citizens, Elections and Parties, Oslo, Universitetsforlaget.

SCHEPS, R. (dir.) (1998). La fabrication de la mort, Institut Synthélabo.

SCHIFFINO, N. (2005). "Comment démocratiser la régulation publique des enjeux techniques", dans P. NIHOUL et S. MAHIEU (dir.), La sécurité alimentaire et la réglementation des OGM. Perspectives nationale, européenne et internationale, Bruxelles, Larcier.
SCHIFFINO, N. (2004). «Biotechnologies et démocratie: statut et évolution de l'expertise dans la prise de décision politique», dans S. JACOB et J.-L. GENARD (dir.), Expertise et action publique, coll. "Sociologie politique», Bruxelles, Éditions de l'ULB.

SCHIFFINO, N. (2003). Crises politiques et démocratie en Belgique, Paris, L'Harmattan, coll. «Logiques politiques».

SEILER, D.-L. (2000). Les partis politiques, Paris, Armand Colin.

SMITH, A.T. et R. TATALOVICH (2003). Cultures at War. Moral Conflict in Western Democracies, Petersborough, Broadview Press.

VATICAN (1980). "Déclaration sur l'euthanasie», Actes du Saint-Siège, n ${ }^{\circ} 1790$.

WAUTIER, J. (2000). «L'ouverture du débat sur l'euthanasie au Sénat. Cadre éthique, médical, juridique et politique», Courrier hebdomadaire du CRISP, $\mathrm{n}^{\circ}$ 1672-1673.

\section{Notes}

1. Loi relative à l'euthanasie, 28 mai 2002, publiée au Moniteur Belge le 22 juin 2002.

2. La dichotomie et l'articulation des deux pôles que sont la gestion du bien public et la res privata sont étudiées de longue date en sciences politiques (Parsons, 2003, p. 2-13).

3. Le concept de non-décision relève d'une tradition d'études en science politique (voir Bachrach et Baratz, 1963)

4. Nous n'entrons pas ici dans les détails du lien entre expertise scientifique et prise de décisions politiques mais le lecteur intéressé peut se référer notamment à Schiffino (2005, 2004) ou encore Montpetit et al. (2006).

5. Dans le monde associatif, les groupes qui se sont le plus fortement mobilisés sont l'Association pour le droit de mourir dans la dignité et le Centre d'action laïque. Le Conseil interdiocésain des laïcs a, quant à lui, globalement suivi la position officielle de l'Église (voir par exemple Vatican, 1980). Des associations défendant les droits des personnes handicapées, telles l'ACIH-AAM (mouvement social des personnes malades, valides et handicapées), se sont également positionnées. Au sein du milieu médical, l'Ordre des médecins a édicté des articles dans son code de déontologie et adopté des avis, allant de l'interdit (article 95 à la suite de la réforme du code de déontologie en 1992) à une certaine ouverture (avis du 15 janvier 2000) envers l'euthanasie.

6. En 1958, l'adoption d'un Pacte scolaire apporte une solution au conflit entre cléricaux et anticléricaux. S'il ne supprime pas les causes du clivage philosophico-religieux, il en anesthésie les effets, contribuant à une sécularisation durable de la vie politique.

7. Le refus de traitement est autorisé par la loi du 22 août 2002 relative aux droits du patient. Cette autorisation a été réaffirmée par la Commission fédérale de contrôle et d'évaluation de l'euthanasie, notamment dans son Deuxième rapport aux chambres législatives (années 2004 et 2005).
8. Le souhait de mourir chez soi reste une demande fréquemment exprimée. L'euthanasie est alors pratiquée par un médecin généraliste. Un problème soulevé par ce type de pratiques était lié à la difficulté pour le médecin d'obtenir la substance létale en officine publique. Les modalités pratiques ont été réglementées par la loi du 10 novembre 2005 (Moniteur Belge du 13 décembre 2005) qui complète la loi du 28 mai 2002 relative à l'euthanasie. Des dispositions concernant le rôle du pharmacien ainsi que l'utilisation et la disponibilité des substances euthanasiantes figurent à l'article 3 bis modifié. Au sujet de l'actualisation de la loi, voir aussi Delfosse et Bert (2005, p. 535).

9. Cette enquête a été menée par une association de consommateurs dans quatre pays d'Euroconsumers: Belgique, Italie, Espagne, Portugal. Seule la Belgique dispose d'une loi dépénalisant l'euthanasie. Cette enquête comporte deux volets: le premier concerne les soins de fin de vie et les soins palliatifs tandis que le second concerne l'euthanasie et les actes de fin de vie. Le public cible était composé de 1253 proches parents, 865 médecins (généralistes et spécialistes) et 967 infirmiers. Dans notre article sont pris en considération les résultats du deuxième volet de l'enquête.

10. Le moment naturel est le moment estimé de la mort du patient si celui-ci ne subit aucun acharnement thérapeutique.

11. Analysant les résultats d'une enquête menée en 2001-2002 sur les décisions médicales de fin de vie, les auteurs émettent l'hypothèse que la sédation profonde peut parfois être considérée comme une alternative pour mettre un terme à la vie mais sans complications légales.

12.Les documents sont rédigés en français ou en néerlandais selon qu'ils concernent les actes pratiqués respectivement au Sud ou au Nord de la frontière linguistique belge, conformément à la structuration de la Belgique en un État fédéral.

13. Les auteurs expliquent que le patient ou sa famille ne sont pas toujours impliqués dans le processus de décision de fin de vie.

14. Code de la Santé Publique, art. L. 1111-10 inséré par la loi $n^{0}$ 2005-370 du 22 avril 2005.

15.Idem, et art. L. 1110-10 inséré par la loi $\mathrm{n}^{\circ} 2002-303 \mathrm{du} 4$ mars 2002.

16. Loi relative aux soins palliatifs du 14 juin 2002, publiée au Moniteur Belge du 26 octobre 2002 .

17. Proposition de loi modifiant la loi du 28 mai 2002 relative à l'euthanasie, Sénat, Session 2003-2004, document no 3-804/1 du 7 juillet 2004.

18. Voir aussi à ce sujet la Proposition de loi complétant, en ce qui concerne les mineurs, la loi du 28 mai 2002 relative à l'euthanasie, Chambre des Représentants, document $n^{\circ} 512553 / 001$ du 15 juin 2006.

19. Proposition de loi modifiant l'article 4 de la loi du 28 mai 2002 relative à l'euthanasie, Sénat, session 2005-2006, document $n^{\circ}$ 3-1485/1 du 14 décembre 2005 . 